\title{
Mania develops in late adolescence
}

Published online: 12 June 2012

(C) Springer Healthcare 2012

MedWire News: There is an increasing prevalence of bipolar disorder over adolescence, with late adolescence the peak period for the development of mania, research shows.

From ages 13-14 years to 17-18 years, the rate of mania doubled in a large sample of adolescents, an increase in rates comparable with adults, according to Kathleen Merikangas (National Institutes of Health, Bethesda, Maryland, USA) and colleagues.

Given that more than one in five children with bipolar disorder had made at least one suicide attempt, the findings are "quite alarming in light of the young age and community setting of this sample," they remark.

The results are taken from a cross-sectional survey of more than 10,000 adolescents aged 13-18 years old. Published in the Archives of General Psychiatry, the report highlights the prevalence of mania with and without depressive episodes in US adolescents.

Using DSM-IV criteria, 2.5\% of youth met the criteria for lifetime bipolar I or II disorder, and $1.7 \%$ met the criteria for mania alone. Within the last 12 months, $2.2 \%$ of the study sample met the criteria for bipolar I and II disorder, and $1.3 \%$ for mania.

Overall, participants aged 17-18 years had a significantly higher rate of mania and major depressive disorder (odds ratio $[\mathrm{OR}]=1.5$ ) and mania alone (OR=2.0) than adolescents aged 1314 years old.

Similarly, those aged 17-18 years old had a twofold higher rate of major depressive disorder (OR 2.1) compared with participants aged 13-14 years old.

Individuals with mania and major depressive disorders had symptoms occur at an earlier age, than those with major depressive disorder alone, and their symptoms were more frequent and severe.

"Other clinical data reinforce that people experiencing both depression and hypomania or mania have higher rates of suicidality," note Merikangas and colleagues.

These results highlight the importance of reconsidering mania as a core feature underlying psychopathology in adolescents, they state.

\section{By MedWire Reporters}

\section{Reference}

Arch Gen Psychiatry 2012; Advance online publication 